\title{
Small cell lung cancer transformation and the T790M mutation: A case report of two acquired mechanisms of TKI resistance detected in a tumor rebiopsy and plasma sample of EGFR-mutant lung adenocarcinoma
}

\author{
GRETA ALI ${ }^{1}$, ROSSELLA BRUNO ${ }^{2}$, MIRELLA GIORDANO $^{2}$, IRENE PREDILETTO ${ }^{3}$, LETIZIA MARCONI ${ }^{3}$, \\ SIMONETTA ZUPO ${ }^{4}$, FRANCO FEDELI ${ }^{5}$, ALESSANDRO RIBECHINI ${ }^{6}$, \\ ANTONIO CHELLA $^{3}$ and GABRIELLA FONTANINI ${ }^{2,7}$ \\ ${ }^{1}$ Unit of Pathological Anatomy, University Hospital of Pisa; ${ }^{2}$ Department of Surgical, Medical and Molecular Pathology and \\ Critical Area, University of Pisa, I-56126 Pisa; ${ }^{3}$ Unit of Pneumology, University Hospital of Pisa, I-56124 Pisa; \\ ${ }^{4}$ Unit of Molecular Diagnostics, Institute of Hospitalization and Scientific Care, National Institute for Cancer Research, \\ I-16132 Genova; ${ }^{5}$ Unit of Pathological Anatomy, Sant'Andrea Hospital, I-19124 La Spezia; \\ ${ }^{6}$ Endoscopic Section of Pneumology, University Hospital of Pisa, I-56124 Pisa; \\ ${ }^{7}$ Program of Pleuropulmonary Pathology, University Hospital of Pisa, I-56126 Pisa, Italy
}

Received May 10, 2016; Accepted July 28, 2016

DOI: $10.3892 / \mathrm{ol} .2016 .5193$

\begin{abstract}
The present study describes the case of a 45-year-old man diagnosed with metastatic lung adenocarcinoma, which harbored a deletion within exon 19 of the epidermal growth factor receptor $(E G F R)$ gene. The patient was subsequently treated with gefitinib (250 mg/day orally from May 2013 to March 2014), but developed acquired resistance to the drug following 11 months of treatment. Tumor burden molecular analysis was performed on a tumor rebiopsy and plasma sample, and histological analysis was also performed on the tumor rebiopsy. A small cell transformation retaining the original EGFR mutation was detected in the tumor rebiopsy, while the T790M mutation together with the activating ex19del mutation were identified only in the plasma sample. The patient was treated with cytotoxic chemotherapy (off-label schedule with epirubicin $80 \mathrm{mg} / \mathrm{mq}$ and paclitaxel $160 \mathrm{mg} / \mathrm{mq}$ every 21 days for 6 cycles) and radiation $(50.4 \mathrm{~Gy}$ administered in 28 fractions of $1.8 \mathrm{~Gy}$ once daily for 5.5 weeks) specific for small cell lung cancer, and may also have benefitted from treatment with a third generation T790M-specific EGFR-TKI. To better describe the mechanisms of resistance to TKI inhibitors and to optimize therapeutic
\end{abstract}

Correspondence to: Professor Gabriella Fontanini, Department of Surgical, Medical and Molecular Pathology and Critical Area, University of Pisa, Via Savi 10, I-56126 Pisa, Italy

E-mail: gabriella.fontanini@med.unipi.it

Key words: lung adenocarcinoma, epidermal growth factor receptor/tyrosine kinase inhibitor-acquired resistance, small cell carcinoma transformation, epidermal growth factor receptor T790M mutation, circulating free tumor DNA regimens, the simultaneous analysis of tumor biopsies and circulating tumor DNA should be considered.

\section{Introduction}

Patients with non-small cell lung cancer (NSCLC) harboring epidermal growth factor receptor $(E G F R)$ activating mutations usually undergo treatment with EGFR-tyrosine kinase inhibitors (EGFR-TKIs) (1). However, despite the initially positive impact of such inhibitors, nearly all patients develop resistance following 8-16 months of treatment (2). Several acquired-resistance mechanisms have been identified; the most common is the EGFR T790M secondary mutation within exon 20 , observed in 50-65\% of resistant disease (3). Other resistant mechanisms are based on the bypassing of transmembrane kinase receptors and include amplification of the $c-M E T$ oncogene (4), overexpression and mutation of ErbB2 (5) and upregulation of Axl, which may activate Akt, mitogen activated protein kinase or nuclear factor $-\kappa \mathrm{B}$ signaling (6). Less common mechanisms of TKI resistance mechanisms may include small cell histological transformation (7) and transition to a mesenchymal phenotype $(8,9)$.

The frequency and possible overlay of these mechanisms has not yet been elucidated. Currently, the use of mutant-selective inhibitors of EGFR and the combination of EGFR-TKIs with drugs inhibiting a specific pathway of resistance represent a possible clinical approach to overcome EGFR-TKI resistance (10). Therefore, rebiopsies of growing tumors during clinical progression are considered critical to characterize the mechanisms of acquired resistance to EGFR-TKIs for therapeutic and prognostic reasons $(3,11)$.

However, a single tumor rebiopsy may not be representative of the dominant characteristics of the tumor due to the well-known intratumor heterogeneity of resistant mechanisms 
in lung cancer (7). Recently, the analysis of blood samples has been suggested to reflect the dominant properties of tumors, and the detection of T790M in plasma may qualify patients as candidates for treatment with a third generation EGFR-TKI(12).

The present study describes a case of tumor heterogeneity of acquired resistance following EGFR-TKI treatment failure.

\section{Case report}

In March 2013, a 45-year-old man with no history of smoking was subjected to medical examination at the Unit of Pneumology, University Hospital of Pisa (Pisa, Italy) due to a persistent cough. Computed tomography $(\mathrm{CT})$ revealed a $3.8-\mathrm{cm}$ right middle lobe mass and bilateral nodules, with the largest measuring $8 \mathrm{~mm}$. In addition, enlarged right hilar and subcarinal lymph nodes were observed. A positron emission tomography (PET) scan exhibited increased fluorodeoxyglucose uptake in the lung lesions and the lymphadenopathy. The patient was reported to have a single 13-mm hepatic metastatic lesion and several bone lesions (in the thighbone, the scapula, and C4 and D10 lamina).

Broncoscopic biopsy was performed. The obtained tissue was formalin-fixed, paraffin-embedded and cut into $5 \mu \mathrm{m}$ sections, which were subsequently stained with hematoxylin and eosin (Fig. 1A). Histological examination of the biopsy identified the presence of adenocarcinoma, which was confirmed by further immunohistochemical examination demonstrating strong nuclear expression of thyroid transcription factor 1 (TTF1) and negative expression of p63 (Fig. 1A and B). Analysis of EGFR mutational status, determined by Sanger sequencing, indicated an EGFR exon 19 deletion.

In April 2013, the patient began treatment with the TKI inhibitor gefitinib (250 mg/day orally for 11 months) and zoledronic acid ( $4 \mathrm{mg}$ every 28 days intravenously for 11 months) for the T4N2M1b adenocarcinoma. In May 2013, the patient also received radiation ( $27 \mathrm{~Gy}$ administered in a single fraction) to the osteoblastic lesion of the thighbone. After 1 month, a CT/ PET scan was performed and indicated a significant decrease in the size of the right middle lobe mass, the bilateral nodules, and the hilar and subcarinal lymphadenopathy. CT/PET scans performed in September and December 2013 were indicative of stable disease. However, by March 2014, disease progression was observed. Therefore, the following treatment regimen was initiated in April 2014: 6 cycles of $200 \mathrm{mg} / \mathrm{m}^{2}$ paclitaxel, 6 AUC carboplatin and $15 \mathrm{mg} / \mathrm{m}^{2}$ bevacizumab administered intravenously every 21 days, followed by 5 cycles of maintenance therapy with $15 \mathrm{mg} / \mathrm{m}^{2}$ bevacizumab administered intravenously every 21 days until December 2014. By that time, the disease had progressed with slight growth of the right middle lobe lesion and a right inferior paraesophageal lymphadenopathy $(11 \times 13 \mathrm{~mm})$ had appeared. Furthermore, a magnetic resonance imaging scan of the brain identified a frontal lobe lesion.

The patient subsequently underwent radiation (whole-brain radiation of 30 Gy administered in 10 fractions of 3 Gy for 2 weeks) to the whole brain and received oral third-line therapy with erlotinib (150 $\mathrm{mg} /$ day) for 4 months and intravenous bevacizumab $\left(15 \mathrm{mg} / \mathrm{m}^{2}\right)$ every 21 days for 6 cycles.

In April 2015, a full-body CT scan was performed and identified a new right middle lobe mass, a slight increase in the right lower lobe nodule and a marked dimensional growth of the right inferior paraesophageal lymphadenopathy (22x22 vs. $11 \times 13 \mathrm{~mm}$ previously).

In June 2015, the patient was subjected to a chest CT scan that documented a further increase in the size of the primitive right lower lobe mass and the right inferior paraesophageal lymphadenopathy ( $35 \times 28$ vs. $22 \times 22 \mathrm{~mm}$ previously). Mutational analysis of the EGFR gene was performed on circulating free tumor (cft) DNA purified from $4 \mathrm{ml}$ plasma using the Easy ${ }^{\circledR}$ EGFR Real-time PCR kit (Diatech Pharmacogenetics SRL, Jesi, Italy) and the Myriapod ${ }^{\circledR}$ Lung Status kit with Sequenom MassARRAY ${ }^{\circledR}$ technology (Diatech Pharmacogenetics SRL) according to the manufacturer's protocol. In addition to the original EGFR exon 19 deletion, cftDNA analysis identified a secondary resistant mutation, T790M, occurring in EGFR exon 20.

In July 2015, a CT-guided biopsy and fine-needle aspiration of the right inferior paraesophageal lymphadenopathy indicated an emergent small cell morphology (Fig. 1C). Further immunohistochemical examination for TTF1 (mouse monoclonal primary antibody; clone 8G7G3/1; ready-to-use; \#790-438), cluster of differentiation 56 (mouse monoclonal primary antibody; clone 123C3; ready-to-use; \#790-4465) chromogranin (mouse monoclonal primary antibody; clone LK2H10; ready-to-use; \#760-2519) and cytokeratin-pan (mouse monoclonal primary antibody; clone AE1; ready-to-use; \#760-2521) (Ventana Medical Systems, Inc., Tucson, AZ, USA) expression was positive (Fig. 1D).

Molecular characterization of the EGFR mutational status of the rebiopsy, performed using the same methods employed for cftDNA analysis, detected the presence of an exon 19 deletion alone.

To more effectively assess the presence of the T790M mutation, further molecular analyses were performed on the cftDNA and rebiopsy using the RainDrop ${ }^{\circledR}$ Digital PCR system (Diatech Pharmacogenetics SRL) specifically for T790M, which is more sensitive than quantitative polymerase chain reaction and Sequenom MassARRAY technology (13). The PCR thermal cycling conditions were as follows: Polymerase activation step at $95^{\circ} \mathrm{C}$ for $10 \mathrm{~min}$; denaturation step at $95^{\circ} \mathrm{C}$ for $15 \mathrm{sec}$ for 50 cycles; annealing-extension step at $59^{\circ} \mathrm{C}$ for $60 \mathrm{sec}$ for 50 cycles; incubation step at $98^{\circ} \mathrm{C}$ for $10 \mathrm{~min}$; further incubation step at $12^{\circ} \mathrm{C}$ for $10 \mathrm{~min}$; and a final hold at $4^{\circ} \mathrm{C}$ for no more than $1 \mathrm{~h}$ until digital analysis. The T790M mutation was detected following liquid biopsy (Fig. 2) with a mutant allele prevalence of $7.15 \%$, which was consistent with the previous results.

Due to the SCLC transformation, the patient began fourth-line chemotherapy, which consisted of epirubicin $\left(80 \mathrm{mg} / \mathrm{m}^{2}\right)$ and paclitaxel $\left(160 \mathrm{mg} / \mathrm{m}^{2}\right)$ administered intravenously every 21 days. Re-evaluation with a full-body CT scan following 2 cycles of epirubicin plus paclitaxel identified a significant dimensional decrease in the right inferior paraesophageal lymphadenopathy (15x20 vs. $35 \times 28 \mathrm{~mm}$ previously) and of the hepatic metastatic lesion ( 8 vs. $10 \mathrm{~mm}$ ). Stability of the lung right lobe lesion, the bilateral satellite nodules, the hilar and subcarinal lymphadenopathy, and the osteoblastic bone lesions were all observed.

At present, the patient is completing 6 cycles of chemotherapy with epirubicin plus paclitaxel and is waiting to begin radiotherapy on the hepatic metastatic lesion. Furthermore, due to the presence of non-responsive lesions and according to the T790M mutation detected in cftDNA, the patient is 

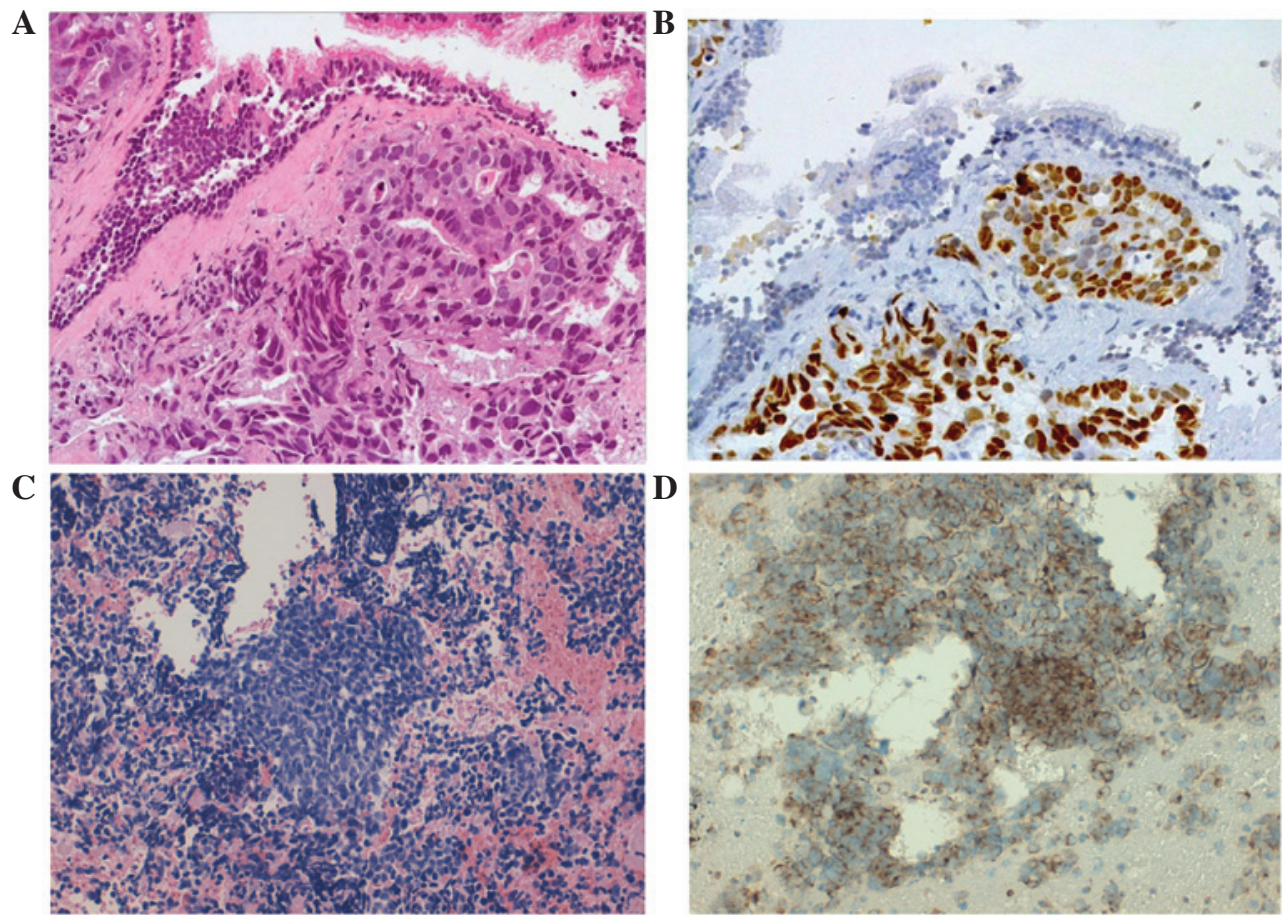

Figure 1. (A) Presence of lung adenocarcinoma in the bronchial biopsy (staining, hematoxylin and eosin). (B) Immunohistochemical staining of thyroid transcription factor 1 demonstrating strong nuclear expression in neoplastic cells. (C) Presence of small cell lung cancer in a computed tomography-guided biopsy (staining, hematoxylin and eosin) (D) with strong cytoplasmic immunoreactivity for chromogranin. All sections were analyzed at a magnification of x20.

A

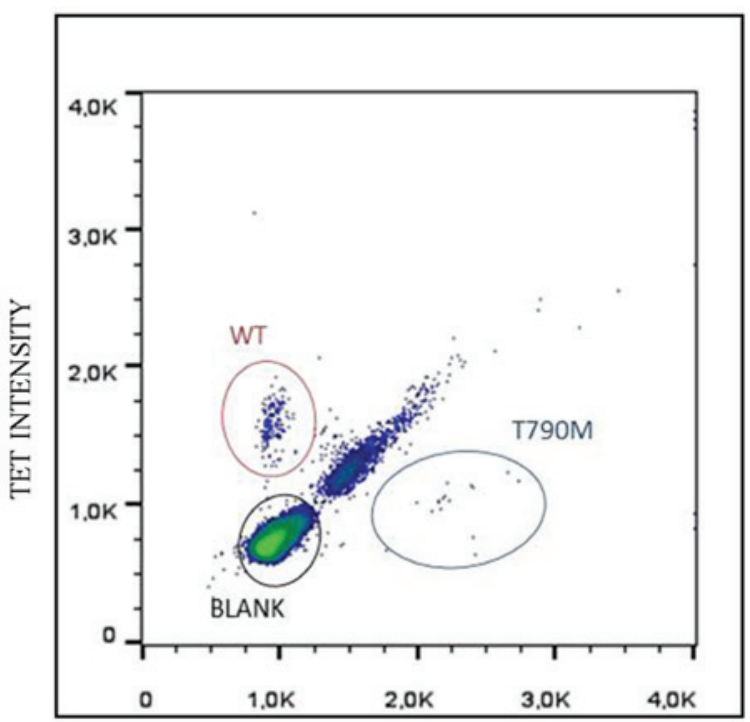

FAM INTENSITY
B

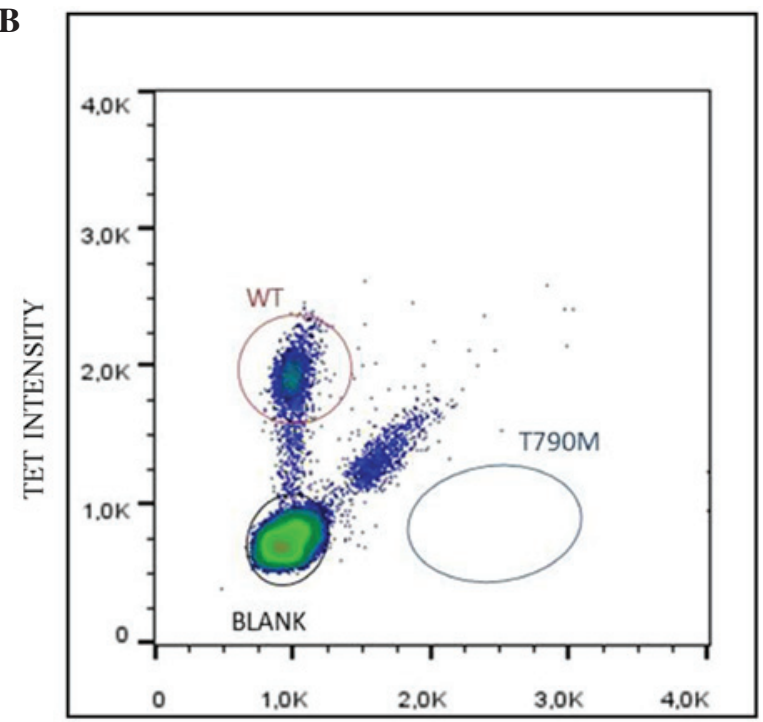

FAM INTENSITY

Figure 2. Analysis of the EGFR T790M mutation in the (A) cftDNA and (B) tumor rebiopsy using a polymerase chain reaction assay specifically for T790M. The WT (detected using a TET-labelled probe) and mutant T790M (detected using a FAM-labelled probe) populations are circled in each panel. The EGFR T790M mutation was observed only in the cftDNA sample with a count number of 13 and was not detected in the corresponding rebiopsy. The counts for the WT allele were 169 and 2459 in the cftDNA and tumor rebiopsy, respectively. EGFR, epidermal growth factor receptor; TET, tetrachloro fluorescein; FAM, 6-carboxyfluorescein; cft, circulating free tumor; WT, wild-type.

currently being considered for treatment with a third generation TKI. Written informed consent for the publication of the current study was obtained from the patient.

\section{Discussion}

Acquired resistance to TKI therapy may be due to multiple biological mechanisms $(2,8,13)$. The use of mutant-selective inhibitors of EGFR, in addition to a combination of treatments and multi-targeting drugs, constitutes current clinical approaches for overcoming EGFR-TKI resistance in NSCLC $(14,15)$. Therefore, the characterization of all the molecular resistant mechanisms occurring in the same patient is crucial to define a more effective therapeutic regimen.

The present study described the case of a patient diagnosed with metastatic lung adenocarcinoma harboring a deletion 
within exon 19 of EGFR, who developed two resistant mechanisms against TKI: A small cell histological transformation and the EGFR T790M mutation. Small cell transformation and the T790M mutation are clinically relevant mechanisms of drug resistance (7), however, their interaction and overlapping is not yet fully understood.

The coexistence of SCLC transformation and the EGFR T790M mutation in response to EGFR-TKI therapy has been described in various studies $(3,16-19)$; however, the majority of these refer to autopsy cases or to cases where a direct analysis of repeat tumor biopsies was possible.

To the best of our knowledge, in all previously reported cases, SCLC metastatic lesions harbored only the EGFR activating mutation, while in the current case the adenocarcinoma metastases harbored the T790M mutation together with the original EGFR mutation. Only one study, by $\mathrm{Yu}$ et al (3), describes SCLC transformation and the EGFR T790M mutation occurring in the same tumor rebiopsies in response to EGFR-TKIs.

The current study and published literature suggest that SCLC and adenocarcinoma components arise from the same origin due to them both possessing the identical activating mutation in EGFR, reflecting the importance of tumor heterogeneity in acquired resistance to TKIs $(16-18,20)$. In the present study, molecular and histological analyses of the tumor rebiopsy and molecular analysis of the corresponding blood sample were performed, enabling a clearer image of TKI resistance. If only the rebiopsy or the cftDNA had been analyzed, coexistence of the two resistant mechanisms would not have been detected.

In comparison with previous cases, the patient described in the present study is currently alive and only one rebiopsy was performed. The plasma sample highlighted tumor properties that were unable to be identified in tissue, therefore making it possible to improve the efficacy of the therapeutic regimen.

In conclusion, on the basis of the histological analysis of the paraesophageal lymph nodes, the current patient was treated for neuroendocrine carcinoma and experienced a clinical response for lymphadenopathy and hepatic metastasis. All other neoplastic lesions, including the primary tumor, did not respond to this treatment. This lack of responsiveness may be due to the heterogeneous EGFR molecular status of the tumor, confirmed by the presence of the T790M mutation in cftDNA, which most likely reflects unresponsive lesions. As such, the patient may benefit from a third generation T790M-specific EGFR-TKI. Therefore, the present study underlines the importance of performing, whenever possible, tumor biopsies following the development of TKI resistance, together with monitoring drug resistance by plasma-based assessments of cftDNA.

\section{References}

1. Mitsudomi T, Morita S, Yatabe Y, Negoro S, Okamoto I, Tsurutani J, Seto T, Satouchi M, Tada H, Hirashima T, et al: Gefitinib versus cisplatin plus docetaxel in patients with non-small-cell lung cancer harbouring mutations of the epidermal growth factor receptor (WJTOG3405): An open label, randomised phase 3 trial. Lancet Oncol 11: 121-128, 2010.

2. Pao W, Miller VA, Politi KA, Riely GJ, Somwar R, Zakowski MF, Kris MG and Varmus H: Acquired resistance of lung adenocarcinomas to gefitinib or erlotinib is associated with a second mutation in the EGFR kinase domain. PLoS Med 2: e73, 2005.
3. Yu HA, Arcila ME, Rekhtman N, Sima CS, Zakowski MF, Pao W, Kris MG, Miller VA, Ladanyi M and Riely GJ: Analysis of tumor specimens at the time of acquired resistance to EGFR-TKI therapy in 155 patients with EGFR-mutant lung cancers. Clin Cancer Res 19: 2240-2247, 2013.

4. Cappuzzo F, Jänne PA, Skokan M, Finocchiaro G, Rossi E, Ligorio C, Zucali PA, Terracciano L, Toschi L, Toschi L, et al: MET increased gene copy number and primary resistance to gefitinib therapy in non-small-cell lung cancer patients. Ann Oncol 20: 298-304, 2009.

5. Takezawa K, Parazzoli V, Arcila ME, Nebhan CA, Song X, de Stanchina E, Ohashi K, Janjigian YY, Spitzler PJ, Melnick MA, et al: HER2 amplification: A potential mechanism of acquired resistance to EGFR inhibition in EGFR-mutant lung cancers that lack the second-site EGFR T790M mutation. Cancer Discov 2: 922-933, 2012.

6. Zhang Z, Lee JC, Lin L, Olivas V, Au V, LaFramboise T, Abdel-Rahman M, Wang X, Levine AD, Rho JK, et al: Activation of the AXL kinase causes resistance to EGFR-targeted therapy in lung cancer. Nat Genet 44: 852-860, 2012.

7. Sequist LV, Waltman BA, Dias-Santagata D, Digumarthy S, Turke AB, Fidias P, Bergethon K, Shaw AT, Gettinger S, Cosper AK, et al: Genotypic and histological evolution of lung cancers acquiring resistance to EGFR inhibitors. Sci Transl Med 3: 75ra26, 2011.

8. Huang L and Fu L: Mechanisms of resistance to EGFR tyrosine kinase inhibitors. Acta Pharm Sin B 5: 390-401, 2015.

9. Lim J and Thiery JP: Epithelial-mesenchymal transitions: Insights from development. Development 139: 3471-3486, 2012.

10. Janjigian YY, Smit EF, Groen HJ, Horn L, Gettinger S, Camidge DR, Riely GJ, Wang B, Fu Y, Chand VK, et al: Dual inhibition of EGFR with afatinib and cetuximab in kinase inhibitor-resistant EGFR-mutant lung cancer with and without T790M mutations. Cancer Discov 4: 1036-1045, 2014.

11. Cross DA, Ashton SE, Ghiorghiu S, Eberlein C, Nebhan CA, Spitzler PJ, Orme JP, Finlay MR, Ward RA, Mellor MJ, et al: AZD9291, an irreversible EGFR, TKI, overcomes T790M-mediated resistance to EGFR inhibitors in lung cancer. Cancer Discov 4: 1046-1061, 2014.

12. Ishii H, Azuma K, Sakai K, Kawahara A, Yamada K, Tokito T, Okamoto I, Nishio K and Hoshino T: Digital PCR analysis of plasma cell-free DNA for non-invasive detection of drug resistance mechanisms in EGFR mutant NSCLC: Correlation with paired tumor samples. Oncotarget 6: 30850-30858, 2015.

13. Bordi P, Del Re M, Danesi R and Tiseo M: Circulating DNA in diagnosis and monitoring EGFR gene mutations in advanced non-small cell lung cancer. Transl Lung Cancer Res 4: 584-597, 2015.

14. Jorge SE, Kobayashi SS and Costa DB: Epidermal growth factor receptor (EGFR) mutations in lung cancer: Preclinical and clinical data. Braz J Med Biol Res 47: 929-939, 2014.

15. Piotrowska Z, Niederst MJ, Karlovich CA, Wakelee HA, Neal JW, Mino-Kenudson M, Fulton L, Hata AN, Lockerman EL, Kalsy A, et al: Heterogeneity underlies the emergence of EGFRT790 wild-type clones following treatment of T790M-Positive cancers with a third-generation EGFR inhibitor. Cancer Discov 5: 713-722, 2015.

16. Morinaga R, Okamoto I, Furuta K, Kawano Y, Sekijima M, Dote K, Satou T, Nishio K, Fukuoka M and Nakagawa K: Sequential occurrence of non-small cell and small cell lung cancer with the same EGFR mutation. Lung Cancer 58: 411-413, 2007.

17. Furugen M, Uechi K, Hirai J, Aoyama H, Saio M, Yoshimi N, Kinjo T, Miyagi K, Haranaga S, Higa F, et al: An autopsy case of two distinct, acquired drug resistance mechanisms in epidermal growth factor receptor-mutant lung adenocarcinoma: Small cell carcinoma transformation and epidermal growth factor receptor T790M mutation. Intern Med 54: 2491-2496, 2015.

18. Popat S, Wotherspoon A, Nutting CM, Gonzalez D, Nicholson AG and O'Brien M: Transformation to 'high grade' neuroendocrine carcinoma as an acquired drug resistance mechanism in EGFR-mutant lung adenocarcinoma. Lung Cancer 80: 1-4, 2013.

19. Fallet V, Ruppert AM, Poulot V, Lacave R, Belmont L, Antoine M, Cadranel J, Wislez M and Lavolé A: Secondary resistance to erlotinib: Acquired T790M mutation and small-cell lung cancer transformation in the same patient. J Thorac Oncol 7: 1061-1063, 2012.

20. Costa DB and Kobayashi SS: Whacking a mole-cule: Clinical activity and mechanisms of resistance to third generation EGFR inhibitors in EGFR mutated lung cancers with EGFR-T790M. Transl Lung Cancer Res 4: 809-815, 2015. 